\title{
Astronomical Fixes Without an Assumed Position
}

\author{
Bernard Spencer
}

A. Chiesa and R. Chiesa ${ }^{1}$ have drawn attention to a method of obtaining an astronomical fix without an assumed position. The present writer further encourages use of the principle, and the writing of programs for the purpose on small computers.

1. INTRODUCTION. On learning about the intercept method many years ago, the writer was impressed by its ingenuity, simplicity and elegance, compared with the older methods where latitude and longitude are determined separately. It is, of course, a rho-rho method in which the distances from the geographical positions (g.p.) of the bodies observed are given by their zenith distances. To obtain a fix in astronavigation, generally two bodies are observed, giving two position circles each centred on its g.p. These circles intersect at two points, either of which can be the observer's position in theory. In the method of plotting used, straight lines represent small parts of the circles, and the two intersection points are not seen. The ambiguity is overcome because the navigator has to use a DR or 'assumed position ' to start from on his chart, and it is taken for granted that this position will be nearer to the correct intersection than to the other one. In the real world of navigation this will almost always be true, because the distance between the two intersections is large in most cases. Azimuth observations can be used as a check.

This conventional method is very successful, without the need to observe a third body to remove ambiguity, unlike the case of some radio rho-rho systems when the distances may be much smaller. This is fortunate for the astro-navigator, who may not be able to make a third observation because of poor visibility or for other reasons.

Nevertheless, use of an assumed position when it is not a mathematical requirement seems somewhat incongruous, and the plotting an unnecessary feat if done on a chart in a small boat. While the astro-navigator may seldom be troubled about the need for a DR position, some might prefer, as the writer does, not to use one in his fixing method, and to have the two intersections presented for him to choose the correct one. No doubt many people over the years will have realized that the two intersections could be calculated mathematically without an assumed position, but have been deterred by the amount of work involved, until the advent of pocket computers.

2. GEOMETRY. The geometry is simple, as shown in Fig. I. Broadly, there are two ways of calculating the intersections $\mathrm{P}(\mathrm{I})$ and $\mathrm{P}(2)$. In one of these, after finding the g.p. of each body from the almanac and observation time, the equations of the position circles are found in a suitable system of coordinates, and are solved simultaneously to find the intersections.

The other way, used by the writer, is to calculate the length of the great circle joining the two g.p.s A and B (Fig. I), by any of the common trigonometrical methods. Since $A-P(1)$ is the zenith distance of $A$ and is known, and similarly $B-P(1)$ is known, three sides of the triangle $A B P(1)$ are known and all the angles can be found. Then with the latitude and longitude of $A$ and $B$, and the sides and angles known, another triangle $\mathrm{N}$ (Pole), $A, P(1)$ can be set up and solved to find the latitude and longitude of $P(1)$.

The other intersection point $P(2)$ must be the mirror image of $P(1)$ on the other side 


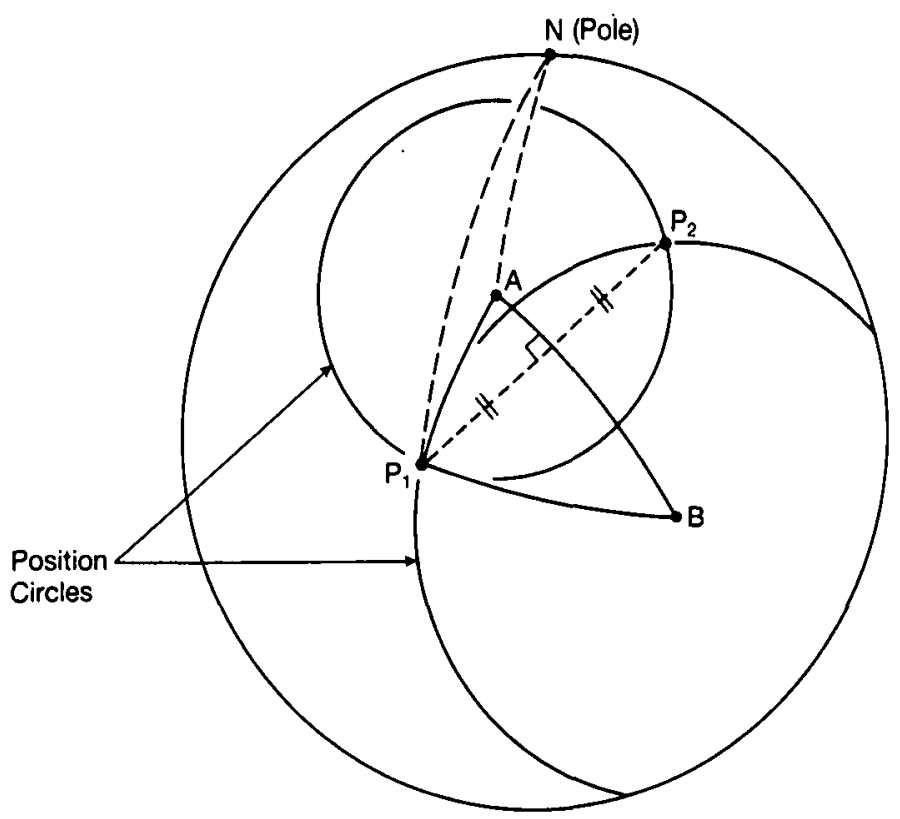

Fig. 1. Determination of $P_{1}$ and $P_{2}$.

$P_{1}-P_{2}$ is the common chord of the position circles.

of the great circle joining $A$ and $B$, and is found easily, as the common chord $P(1)-P(2)$ of the position circles crosses $A-B$ at right angles, and is bisected by $A-B$.

Naturally, whatever formulae are chosen to solve the problem, checks are needed to avoid errors due to any ambiguities, or when unusual values are entered such as when straddling the Date Line, and so on. The writer often uses haversines in trigonometrical calculations instead of simpler functions to minimize the number of possible ambiguities, as the haversine is positive and increases continuously as the angle goes from $\circ$ to 180 degrees. Other formulae are given in Notes and References 1 .

3. COMPUTER PROGRAM. In 1987, the writer decided to produce a program for a pocket computer to give the intersections without an assumed position, as outlined above. It so happened that the first requirement was for a program to be used for the accurate determination of position on land. ${ }^{2}$ This was eventually written for the Sharp PC 1500 Pocket Computer (equivalent to Radio Shack PC2), called ASTRofix, and arranged for easy entry of the Sun and star data as tabulated in the Star Almanac for Land Surveyors (SALS). For the intended land operation, a theodolite would normally be used, but height of eye/temperature/pressure corrections for the sextant are available in the program, as well as theodolite corrections. There are semi-diameter and parallax corrections for the Sun.

A feature of this computer is that it has a fairly accurate quartz clock, and the program allows this to be used for timing observations, but an external chronometer can be used if desired. The program length requires an $8 \mathrm{~K}$ RAM plugged into the back of the computer. A printer is optional.

As an alternative to entering SALS data, the g.p. of stations can be entered in latitude and longitude, and their distances in kilometres, as for a radio rho-rho system. In either case, the coordinates of $\mathrm{P}(1)$ and $\mathrm{P}(2)$ in latitude and longitude are automatically calculated and displayed. The program allows observation of a third body or station to 
be added, to determine whether $\mathrm{P}(\mathrm{I})$ or $\mathrm{P}(2)$ is the correct point, automatically. If there is any doubt, it does not carry out the selection, which is then left to the navigator. For astronomical observations, a third body is seldom required. The program works well.

For use at sea, the SALS can still be used, though it does not give Moon and planet data. Nevertheless, Moon and planet data from another almanac can be used if the navigator is prepared to correct the observation, calculate the g.p., and use the rho-rho entry, which might be useful in an emergency. Of course, for marine navigation it would be better (and not difficult) to modify the program to accept data from the Nautical Almanac or Compact Data. Indeed, a long-term almanac could be written and stored in the computer, as some have done for other kinds of navigation programs. Then, for ordinary navigation accuracy at sea, only altitude, height of eye and name of body need be entered, with the timing automatically read and data processed when the computer ENTER button is pressed.

The program which was written (ASTROFIX) contains a sub-program POLAZ for determination of north on land using a theodolite.

4. CONCLUsion. It is hoped that the excellent paper by Arturo and Raffaele Chiesa' and these notes will lead to much use of this convenient method, and to the writing of suitable programs for various small computers, especially for small-boat use.

\section{NOTES AND REFERENCES}

1 Chiesa, A. and Chiesa, R. (1990). A mathematical method of obtaining an astronomical vessel position. This Journal, 43,125 .

"A cassette tape copy of this PC I500 land program "ASTROFIX" and a copy of the typed program operating notes, can be obtained by Members from the RIN without charge.

KEY WORDS

I. Astro. 2. Computers. 